\title{
Stability and Numerical Simulation of the Liquid Metal Pinch Using the Shallow Water Approximation
}

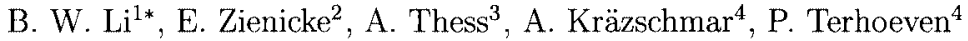 \\ ${ }^{1}$ Key Laboratory of National Education Ministry for Electromagnatic Processing of Materials, POB \\ 314, Northeastern University, Shenyang 110004, China \\ ${ }^{2}$ Institut für Physik, Technische Universität Ilmenau, PF 100565, D-98684 Ilmenau, Germany \\ ${ }^{3}$ Fakultät Maschinenbau, Technische Universität Ilmenau, PF 100565, D-98684 Ilmenau, Germany \\ ${ }^{4}$ Moeller GmbH, Hein Moeller Str. 7-11, D-53115 Bonn, Germany
}

Email: heatli@hotmail.com

\begin{abstract}
In the reference [E. Zienicke, B.W. Li, A. Thess, A. Krätzschmar, P. Terhoeven, Stability Analysis of the Liquid Metal Pinch Using the Shallow Water Approximation, in Fifth International pamir Conference on Fundamental and Applied MHD, Ramatuelle, France-2002, Vol. 1, p.I-51 I-56], a simple physical model for a cylindrical jet of liquid metal passed through by an axial electrical current is introduced and its corresponding mathematical model based on shallow water approximation is deduced. In this paper, the MHD pinch instability is analyzed and compared with results from reference, and hence to guide the application of MHD pinch in construction of electric current switch. Besides this a pseudo spectral method is used to successfully simulate the time evolution of the pinch process. The numerical calculations showed that the nonlinear phase of the pinch process is very short. It starts approximately with a relative perturbation about $1 / 10$ and needs around $0.2 \mathrm{~ms}$ for GaInSn with a radius of $R_{0}=1 \mathrm{~cm}$ and an overload factor of 10 above the critical current density. The shortness of the nonlinear phase is due to a strong self-acceleration of the pinch. If one wants to use the shortness of the nonlinear pinch phase the geometrical design of the switch has to give an initial narrowing of the current path by at least one tenth of the radius.
\end{abstract}

\section{REFERENCES}

1. Zienicke E, Li B W, Thess A, Krätzschmar A, Terhoeven P. Stability analysis of the liquid metal pinch using the shallow water approximation. In: Fifth International pamir Conference on Fundamental and Applied MHD, Ramatuelle, France-2002, 1: I-51 I-56

2. Murty G S. Instability of conducting fluid cylinder due to axial current. Arkiv för Fysik, 1960; 18: 241-250

3. Canuto C, Hussaini M, Quarteroni A, Zang A. Spectral Methods in Fluid Dynamics, Berlin: Springer, 1988 